\title{
Comparison of multisensory and strength training for postural control in the elderly
}

This article was published in the following Dove Press journal:

Clinical Interventions in Aging

18 May 2012

Number of times this article has been viewed

\author{
Fábio Marcon Alfieri' \\ Marcelo Riberto ${ }^{2}$ \\ Lucila Silveira Gatz ${ }^{3}$ \\ Carla Paschoal Corsi \\ Ribeiro ${ }^{4}$ \\ José Augusto Fernandes \\ Lopes $^{4}$ \\ Linamara Rizzo Battistella ${ }^{4}$ \\ 'São Paulo Adventist University \\ Center, São Paulo, ${ }^{2}$ Faculty of Medicine \\ in Ribeirão Preto, University of São \\ Paulo, ${ }^{3}$ Physical Educator, São Paulo, \\ ${ }^{4}$ School of Medicine, Institute of \\ Physical Medicine and Rehabilitation, \\ Clinics Hospital of University of São \\ Paulo, São Paulo, Brazil
}

Objective: The objective of this study was to analyze the efficacy of multisensory versus muscle strengthening to improve postural control in healthy community-dwelling elderly.

Participants: We performed a single-blinded study with 46 community-dwelling elderly allocated to strength (GS, $\mathrm{n}=23 ; 70.18 \pm 4.8$ years 22 women and 1 man) and multisensory exercises groups (GM, $\mathrm{n}=23$; $68.8 \pm 5.9$ years; 22 women and 1 man) for 12 weeks.

Methods: We performed isokinetic evaluations of muscle groups in the ankle and foot including dorsiflexors, plantar flexors, inversion, and eversion. The oscillation of the center of pressure was assessed with a force platform.

Results: The GM group presented a reduction in the oscillation $\left(66.8 \pm 273.4 \mathrm{~cm}^{2}\right.$ to $11.1 \pm 11.6 \mathrm{~cm}^{2} ; P=0.02$ ), which was not observed in the GS group. The GM group showed better results for the peak torque and work than the GS group, but without statistical significance.

Conclusion: Although the GM group presented better results, it is not possible to state that one exercise regimen proved more efficacious than the other in improving balance control.

Keywords: postural control, aging, exercise, multisensory, strengthening

\section{Introduction}

Postural control is a key element in the execution of daily activities (eg, walking, sitting and standing from a chair, etc). This complex function requires input from the afferent receptor systems, vestibular, visual, proprioception, and general exterosensibility, in order to generate a motor response allowing the transition between dynamic and static activities. ${ }^{1-3}$ Aging is associated with a decrease in postural control, which contributes to the increase in falls among the elderly. ${ }^{3-5}$

One of the most common interventions to prevent the consequences (eg, fractures and falls) of decreased balance is physical exercise. Besides promoting better health conditions, physical exercise can improve postural control and reduce the tendency to falls in this population. ${ }^{6,7}$ A growing number of studies have demonstrated this association, ${ }^{8}$ and several exercises have been shown to have some effect on the desired outcome. ${ }^{9}$ In particular, multisensory exercises ${ }^{10-12}$ are characteristically defined by the stimulation of all three afferent systems and the ones that strengthen body muscles. ${ }^{13-16}$ Although the latter exercises are efficacious in restoring muscle mass and strength, when they are performed in isolation, their effects on postural control have not been well defined.

We believe that interventions that assess how different exercises interfere with postural control will allow a better and customized design of therapeutic programs to achieve a reduction in falls..$^{8,15}$ The objective of this study was to analyze the efficacy 
of multisensory versus muscle strengthening to improve postural control in healthy community-dwelling elderly people.

\section{Methods}

\section{Design}

The local ethics committee approved the study, and all participants signed an informed consent form. We chose a randomized, single-blinded controlled trial. The study design and procedure for recruitment have been described previously. ${ }^{17}$ The study was performed in a rehabilitation center for outpatients in the central area of São Paulo, Brazil.

\section{Subjects}

The inclusion criteria were the following: age between 60 and 75 years; body mass index between 20 and 30; and ability to participate in physical activities. The exclusion criteria were the following: regular physical training in the last 3 months; severe clinical musculoskeletal impairments; implanted prosthesis of previous fractures in the axial skeleton or lower limbs; visual impairment without correction; and a recent complaint of dizziness or falls. Furthermore, subjects were discontinued from the study if they failed to participate in at least three consecutive training sessions. Before any intervention, they were evaluated by a physician in order to determine any clinical condition that might hinder participation in the study.

\section{Assessment}

Initially, subjects were assessed with the Timed Up and Go (TUG) test, which requires a subject to stand up, walk $3 \mathrm{~m}$ $(10 \mathrm{ft})$, turn, walk back, and sit down. The time taken to complete the test is strongly correlated with the level of functional mobility.$^{18}$ Afterwards, they were evaluated with a functional balance test using the Berg Balance Scale. This test consists of a range of 14 common tasks that involve both static and dynamic balance, such as reaching, turning, standing, arising, and making transfers. ${ }^{19}$

Subjects were evaluated before and after the interventions with a force platform (AMTI Inc, Watertown, MA). Where the oscillation of the body center of pressure area could be defined, we assumed this parameter to represent the whole body oscillation. ${ }^{9,10}$ After calibration, signs of oscillation of the body were recorded with the platform in the on-leg position. Data were captured with Eva software (Hewlett Packard $^{\circledR}$, Palo Alto, CA), in an acquisition frequency of $100 \mathrm{~Hz}$. The area of movement of the center of pressure was measured in centimeters squared $\left(\mathrm{cm}^{2}\right)$. We used a routine developed in Matlab (version 6.5 ${ }^{\circledR}$; MathWorks, Upper Saddle River, NJ) to process the data. ${ }^{20}$

For the assessment of static balance, subjects remained standing barefoot wearing light clothes on the dominant lower limb with open eyes for 10 seconds. In order to calculate the mean, this procedure was repeated three times sequentially, and the subjects were requested to stare at a fixed point within approximately $1 \mathrm{~m}$ at eye level. Illumination, noise, and temperature were kept constant and comfortable for all subjects and sessions. One assessor was positioned beside the subject in order to provide support in the event of a fall.

Isokinetic evaluation of the ankle was performed with an isokinetic evaluator (Cybex Humac Norm ${ }^{\circledR}$; Computer Sports Medicine, Stoughton, MA). Subjects were positioned supine with the hip fixed in $90^{\circ}$ and knee flexion of $80^{\circ}$, and straps were used to keep the lower limb in a fixed position. The movements performed at the heel and foot joints included dorsiflexion, plantar flexion, invertion, and evertion. ${ }^{21}$

Subjects were familiarized with the equipment during three rehearsals with free angular speed in order to get used to the movement to be tested. In order to calculate the mean, testing was performed in five repetitions at an angular speed of $30^{\circ} / \mathrm{s}$, with concentric contractions of agonists and antagonists alternately though all ranges of motion. A verbal stimulus during the tests was essential to promote a better effort from subjects. Peak torque $(\mathrm{N} / \mathrm{m})$ and total work $(\mathrm{J})$ were recorded.

\section{Interventions}

Subjects were asked not to participate in other physical activities and not to change their daily habits during the study. A computer-generated list randomly allocated the subjects to one of two training programs: strength training (GS) or multisensory training (GM). Both groups were subjected to one hour of training sessions on two different days a week. Subjects in both programs remained in training for 12 weeks. ${ }^{9,12}$

\section{Multisensory exercises program (GM)}

Multisensory intervention emphasized the stimuli to sensory systems. A warm-up period before the activity included short walks and games with balls using the hands or feet. These were followed by stretching exercises for hip muscles, flexors, and extensors of the knee, ankle, and paraspinal muscles. Activities were performed both standing and lying down. Resistance exercises for plantar flexors and dorsiflexors as well as squatting and abdominals were performed against gravity in order to strengthen lower limbs and trunk; three series of ten repetitions were performed for each of these exercises. 
Subjects also performed activities to stimulate the plantar surface and dynamic balance. They were asked to walk forwards, backwards, and sideways, with eyes both open and closed at different speeds and for various distances. Ground surfaces also varied and included mattresses and different densities of foam rubber. The subjects were also challenged by obstacles, such as ropes, cones, and sticks. Balance training was performed with devices often directed to the following activity in physical therapy: according to the subject's ability, he or she might be asked to remain standing on uni- or bipedal support with eyes open or closed. These sensorial challenges lasted from 20 to 30 minutes. Finally, motor coordination training was performed by alternate movements of the upper and lower limbs, different positions of the head and neck, both with and without visual stimuli. ${ }^{12,17}$

\section{Strength exercises program (GS)}

The muscle-strengthening program was performed using six different resistance devices with varying resistance: chest press, rowing, leg press, calves, abdominal, and lumbar extension. These devices were chosen because they load the main muscle groups.

At each device, the subjects were oriented to perform three series of exercises with varying loads: the first series


Figure I Flow of patients through the study. 
of twelve repetitions were performed with a load at $50 \%$ of the maximum, the second (ten repetitions) at $75 \%$ of the maximum. The third series required eight repetitions at the maximum tolerated load. On the first day of training, the subjects did the exercises with no load in order to get used to the movements and posture. This value was estimated by successive trials and was defined as the load compatible with a complete range of motion, without a slow concentric or eccentric contraction, or sustained apnea. This value was then recorded to set new limits for the next session. The load was increased whenever it was possible to complete the last series of eight repetitions. Complaints of pain and breathing discomfort were actively elicited, and the speed of movements was constantly monitored in both concentric and eccentric phases..$^{17,22,23}$

\section{Statistical analysis}

The analysis considered a two-parallel arm design and an $80 \%$ probability of detecting a treatment difference at a two-sided 0.05 significance level. If the true difference between treatments was 1.5 units in TUG, two subjects were added in order to compensate for possible dropouts, which resulted in 46 subjects in the final sample. Data analysis was performed with the statistical package SigmaStat 3.5 for Windows (Systat Software, Point Richmond, CA). We used the principle of intention to deal with possible dropouts, and missing data were input with the mean of the remaining data. This strategy does not change the mean of the sample and prevents reduction of statistical power. However, it reduces the standard deviation and facilitates the identification of statistically significant results. The results were described as means and standard deviations. Comparisons of means at baseline between groups were performed using a twotailed, independent Student's $t$-test or Mann-Whitney $U$ test. Comparisons within the groups after the treatments were performed with a paired Student's $t$-test or Wilcoxon test, according to data distribution. The Mann-Whitney $U$ test was used to test the differences in balance outcome variables between the groups in changes during the follow-up. The significance level was 0.05 .

\section{Results}

From the 69 individuals recruited, 46 were eligible and provided their informed consent to participate. The progress of the participants through the various stages of the study is presented in Figure 1.

There were no statistically significant differences between the groups in terms of the proportion of female and male
Table I General characteristics of participants at baseline $($ Mean $\pm \mathrm{SD})$

\begin{tabular}{llll}
\hline & GS & GM & P \\
\hline N & 23 & 23 & \\
F/M & $22 / I$ & $22 / I$ & \\
Age (years) & $70.2 \pm 4.8$ & $68.8 \pm 5.9$ & $0.47^{*}$ \\
BMl (kg/cm²) & $28.2 \pm 3.3$ & $26.9 \pm 3.5$ & $0.30^{*}$ \\
TUG (s) & $8.8 \pm 2.3$ & $9.1 \pm 1.9$ & $0.53^{*}$ \\
Berg & $55.5 \pm 1.4$ & $55 \pm 1.8$ & $0.5 I^{* *}$ \\
\hline Notes: Values are presented as mean (SD). *Unpaired t-test; **Mann-Whitney test. \\
Abbreviations: BMl, body mass index; F, female; M, male; TUG, Timed Up and \\
Go test; s, seconds; Berg, Berg Balance Scale; GS, group strength; GM, group \\
multisensory; SD, standard deviation.
\end{tabular}

participants, age, and body mass index (BMI). Clinically, the groups were similar in functional terms before the intervention when TUG, Berg (Table 1), and displacement of center of pressure (Table 2) were compared.

Isokinetic parameters in the right (Table 3) and left (Table 4) ankles were very similar before the interventions in both groups, except for total work during invertion of the right ankles. Although the differences were not statistically significant, the mean peak torque and work were less in GS before the intervention. After the intervention, although GS presented with improvement in peak torque in plantar flexion bilaterally and dorsiflexion in the left side, the improvements in total work were not statistically significant. However, GM presented with improvement in peak torque in plantar flexion and dorsiflexion bilaterally and in inversion and eversion on the right side. The total work improvement was statistically significant for plantar flexion and dorsiflexion bilaterally in GM.

The comparisons after treatment between the groups of gains after the intervention in all isokinetic parameters were not statistically significant (Tables 3 and 4).

\section{Discussion}

We compared the benefits of two exercise regimens on postural control of healthy elderly subjects. Although both intervention groups showed statistically significant

Table 2 Displacement of center of pressure $\left(\mathrm{cm}^{2}\right)$ (mean and standard deviation) within and between groups before and after interventions

\begin{tabular}{lllll}
\hline & Before & After & $P$ & $P$ \\
\cline { 3 - 5 } & & & Group $\times$ Time \\
\hline displacement & $62.7 \pm 269.8$ & $16.6 \pm 30.5$ & $0.75^{*}$ & $0.28^{* *}$ \\
$\begin{array}{l}\text { GM } \\
\text { displacement }\end{array}$ & $66.8 \pm 273.4$ & $11.1 \pm 11.6$ & $0.02^{*}$ & \\
\hline
\end{tabular}

Notes: *Wilcoxon; **Mann-Whitney.

Abbreviations: GS, group strength; GM, group multisensory. 
Table 3 Peak torque $(\mathrm{N} / \mathrm{m})$ and total work $(\mathrm{J})$ of isokinetic assessment of right heel and foot movements (mean and standard deviation) at $30 \%$, within and between groups: strength (GS) and multisensory (GM) before and after the interventions

\begin{tabular}{|c|c|c|c|c|c|c|c|}
\hline & \multicolumn{3}{|l|}{ GS } & \multicolumn{3}{|l|}{ GM } & \multirow{2}{*}{$\frac{P}{\text { Group } \times \text { Time }}$} \\
\hline & Before & After & $P$ & Before & After & $P$ & \\
\hline \multicolumn{8}{|l|}{ Peak torque } \\
\hline Plantar flexion & $28.6 \pm 9.0$ & $34.3 \pm 13.4$ & 0.03 & $34.3 \pm 13.9$ & $42.7 \pm 9.5$ & 0.007 & 0.55 \\
\hline Dorsiflexion & $12.4 \pm 4.0$ & $13.7 \pm 5.7$ & 0.32 & $13.6 \pm 4.5$ & $15.7 \pm 3.8$ & 0.01 & 0.50 \\
\hline Inversion & $12.7 \pm 5.2$ & $\mid 4.1 \pm 5.2$ & 0.38 & $14.7 \pm 4.6$ & $17.6 \pm 3.1$ & 0.008 & 0.46 \\
\hline Eversion & $10.2 \pm 4.3$ & $1 \mathrm{I} .2 \pm 4.0$ & 0.51 & $11.3 \pm 3.3$ & $13.3 \pm 3.3$ & 0.007 & 0.61 \\
\hline \multicolumn{8}{|l|}{ Total work } \\
\hline Plantar flexion & $16.5 \pm 5.3$ & $18.8 \pm 7.7$ & 0.08 & $20 \pm 8.9$ & $24.9 \pm 7.2$ & 0.031 & 0.33 \\
\hline Dorsiflexion & $7.6 \pm 2.7$ & $8.2 \pm 4.2$ & 0.4 & $8.0 \pm 2.9$ & $9.7 \pm 2.6$ & 0.002 & 0.29 \\
\hline Inversion & $8.4 \pm 3.6$ & $9.4 \pm 3.4$ & 0.42 & $10.7 \pm 3.8^{*}$ & $11.4 \pm 2.6$ & 0.31 & 0.85 \\
\hline Eversion & $8.3 \pm 4.0$ & $9.5 \pm 3.5$ & 0.39 & $9.3 \pm 3.4$ & $9.8 \pm 2.5$ & 0.47 & 0.65 \\
\hline
\end{tabular}

Note: $* P<0.05$ comparison between GS and GM before the intervention.

improvements in many parameters, and they did somewhat better in the multisensory approach, the differences between the groups were not statistically significant. Thus, although both interventions improved static and dynamic mobility in the healthy elderly subjects, we cannot state that either one is more efficacious.

Static balance is a predictor of independence and risk of falls in the elderly. ${ }^{10,24}$ Similarly, muscle action around the heel is also important because it is responsible for the control of this joint and thus support to the whole body. This outcome was assessed in this study because it is used in situations in which there is little disturbance of balance and the standing surface is stable, which were the conditions in this study. ${ }^{2}$

Although the improvement of body oscillation in the GM group was not significantly greater than in the GS group, we believe the results obtained by the GM group are clinically important because the subjects in this group might have achieved better postural control. ${ }^{25}$ Reduced oscillation of the body center of pressure indicates better postural control, whereas a wide area of oscillation might reflect the deterioration of postural control. ${ }^{2}$ By testing this displacement in a standing position, it was possible to assess the muscle effort to maintain balance, ${ }^{11}$ which was confirmed by the results of the isokinetic evaluation in the GM group. The reduced oscillation of the body center of pressure observed in the GM group is associated with the strengthening documented by the peak torque and work in the isokinetic assessment. This association was also observed in another study that used multisensory exercises three times a week for twelve weeks, in which improved action in gastrocnemius and tibialis anterioris resulted in the same improvement in balance.,12

The elderly lose the ankle strategy for postural control due to the muscle weakness and peripheral sensory loss that occur in aging. Improvement of muscle action in this region, which is the first to be used for maintaining balance, can be a contributing factor in the prevention of falls among the elderly. ${ }^{2,12,26,27}$

Although the results for the two groups were not statistically different, we observed that only the GM group

Table 4 Peak torque $(\mathrm{N} / \mathrm{m})$ and total work $(\mathrm{J})$ of isokinetic assessment of left heel and foot movements (mean and standard deviation) at $30 \%$ s, within and between groups: strength (GS) and multisensory (GM) before and after the interventions

\begin{tabular}{|c|c|c|c|c|c|c|c|}
\hline & \multicolumn{3}{|l|}{ GS } & \multicolumn{3}{|l|}{$\mathbf{G M}$} & \multirow{2}{*}{$P$} \\
\hline & Before & After & $P$ & Before & After & $P$ & \\
\hline \multicolumn{8}{|l|}{ Peak torque } \\
\hline Plantar flexion & $27.2 \pm 8.8$ & $34.1 \pm 7.6$ & 0.001 & $33.5 \pm 15.5$ & $42.0 \pm 11$ & 0.01 & 0.74 \\
\hline Dorsiflexion & $12.9 \pm 3.2$ & $15.5 \pm 3.8$ & 0.001 & $13.7 \pm 3.5$ & $16.8 \pm 3.9$ & 0.002 & 0.71 \\
\hline Inversion & $12.8 \pm 4.5$ & $14.2 \pm 2.7$ & 0.12 & $13.9 \pm 3.5$ & $14.8 \pm 4.7$ & 0.4 & 0.74 \\
\hline Eversion & $11.2 \pm 3.8$ & $12.4 \pm 3.4$ & 0.22 & $13.2 \pm 4.3$ & $14.5 \pm 4.7$ & 0.28 & 0.98 \\
\hline \multicolumn{8}{|l|}{ Total work } \\
\hline Plantar flexion & $16.4 \pm 6.1$ & $18.6 \pm 5.8$ & 0.1 & $19.5 \pm 9.5$ & $24.2 \pm 6.9$ & 0.04 & 0.39 \\
\hline Dorsiflexion & $8.0 \pm 2.4$ & $9.1 \pm 3.1$ & 0.06 & $8.0 \pm 2.5$ & $10.0 \pm 2.6$ & 0.004 & 0.25 \\
\hline Inversion & $9.9 \pm 4.1$ & $10.2 \pm 2.7$ & 0.69 & $10.7 \pm 3.2$ & $10.9 \pm 3.5$ & 0.82 & 0.94 \\
\hline Eversion & $8.9 \pm 3.0$ & $9.2 \pm 3.2$ & 0.68 & $10.5 \pm 3.5$ & $10.6 \pm 3.3$ & 0.93 & 0.84 \\
\hline
\end{tabular}


significantly improved the work of the dorsiflexor and plantar flexor muscles, which are important for the maintenance of static posture. ${ }^{2,12}$ This result led us to believe that the intensity, duration, and frequency of the strengthening exercises performed by the subjects in this study were not adequate to promote changes in the muscular action of the ankle joint.

The fact that multisensory exercises were able to reduce body sway in participants might be because this training provided sensory stimulation of the visual, vestibular, and somatosensory systems through exercises that were performed on different types of surfaces and densities. Furthermore, they used balls, circuits, and other devices that provided stimuli that enhance balance. ${ }^{9-11,25,28,29}$

On the other hand, when used alone, muscle-strengthening exercises seem to have no effect on the improvement of balance in the elderly. This may be due to a decrease in sensory information because these exercises were conducted in a sitting position and not in an orthostatic posture in which the balance is most evident. ${ }^{30,31}$

Although strength exercise is often assumed to be beneficial for balance, one review provided evidence that strength exercise used as an isolated intervention is not uniformly effective in improving balance performance. ${ }^{16}$ Only $22 \%$ of the results of the balance tests examined supported the efficacy of strength exercises as a single modality to improve balance. ${ }^{16}$ Although there are significant differences between groups after the intervention, our results corroborate the literature in that stimuli of the sensory systems are required to achieve gains in postural control. ${ }^{9,16}$

Because it confirmed similar findings in other studies, we believe that the evidence in our study is valid and solid. However, the limitations of this study are the reduced sample size, lack of a comparison group with no training, and the design of the strength exercise programs in terms of load, duration, and frequency performed. These aspects can, however, be used as parameters for new steps in the understanding of the effects of multisensory exercises on postural control.

\section{Conclusion}

Although the group that performed multisensory exercises presented with a significant reduction of body oscillation and expressive improvement in isokinetic parameters on the heels after training, we cannot state that any of the exercise programs was superior for postural control.

\section{Acknowledgments}

The authors thank Leslie Andrews Portes for assistance with the data analysis and the statistics. We also thank Carolina
Rodini, Luana Talita Diniz Ferreira, Gemal Emanuel Pirré for their assistance in the subject recruitment and the training. We acknowledge the contributions of Marcelo Alves Mourão and Adilson Gonçalves in the assessment of the functions of the subjects.

\section{Disclosure}

The authors declare no conflicts of interest in this work.

\section{References}

1. Alexander NB. Postural control in older adults. J Am Geriatric Soc. 1994;42:93-108.

2. Shumway-Cook A, Woollacott M. Motor Control: Theory and practical applications. Baltimore, MD: Williams and Wilkins; 1995.

3. Woollacott M, Shumway-Cook A. Attention and the control of posture and gait: a review of an emerging area of research. Gait Posture. 2002;16(1):1-14.

4. Tinetti ME, Speechley M, Ginter SF. Risk factors for falls among elderly person living in the community. N Engl J Med. 1988;319:701-707.

5. Chang JT, Morton SC, Rubenstein LZ, et al. Interventions for the prevention of falls in older adults: systematic review and meta-analysis of randomized clinical trials. BMJ. 2004;328:1-7.

6. Morey MC, Sloane R, Pieper CF, et al. Effect of physical activity guidelines on physical function in older adults. J Am Geriatr Soc. 2008;56(10):1873-1878.

7. Hubbard RE, Fallah N, Searle SD, Mitnitski A, Rockwood K. Impact of exercise in community-dwelling older adults. PLoS One. 2009;4(7):e6174

8. Bulat T, Hart-Hughes S, Ahmed A, et al. Effect of a groupbased exercise program on balance in elderly. Clin Interv Aging. 2007;2(4):655-660.

9. Howe TE, Rochester L, Jackson A, Banks PMH, Blair VA. Exercise for improving balance in older people. Cochrane Database Syst Rev. 2007;4:CD004963.

10. Rogers ME, Rogers NL, Takeshima N, Islam MM. Methods to asses and improve the physical parameters associated with fall risk in older adults. Prev Med. 2003;36:255-264.

11. Rogers ME, Fernandez JE, Bohlken RM. Training to reduce postural sway and increase functional reach in the elderly. J Occup Rehabil. 2001;11(4):291-298.

12. Alfieri FM, Guirro RRJ, Teodori RM. Postural stability of elderly submitted to multisensorial physical therapy intervention. Electromyogr Clin Neurophysiol. 2010;50(2):113-119.

13. Seguin R, Nelson ME. The benefits of strength training for older adults. Am J Prev Med. 2003;25:141-149.

14. Bruin ED, Murer K. Effect of additional functional exercises on balance in elderly people. Clin Rehabil. 2007;21:112-121.

15. Nitz JC, Choy NL. The efficacy of a specific balance-strategy training program for preventing falls among older people: a pilot randomized controlled trial. Age Ageing. 2004;33:52-58.

16. Orr R, Raymond J, Sigh MF. Efficacy of progressive resistance training on balance performance in older adults. Sports Med. 2008;38(4):317-343.

17. Alfieri FM, Riberto M, Gatz LS, Ribeiro CPC, Lopes JAF, Battistella LR. Functional mobility and balance in community-dwelling elderly submitted to multisensory versus strength exercises. Clin Interv Aging. 2010;5:181-185.

18. Podsiadlo D, Richardson S. The timed "Up and Go": a test of basic functional mobility for frail elderly persons. J Am Geriatr Soc. 1991;39:142-148.

19. Berg KO, Woo-Dauphinee SL, Williams JI, Maki B. Measuring balance in the elderly: validation of an instrument. Can J Public Health. 1992;83(Suppl 2):S7-S11. 
20. Raymakers JA, Samson MM, Verhaar HJJ. The assessment of body sway and the choice the stability parameter(s). Gait Posture. 2005; 21(1):48-58.

21. Imamura M. Avaliação Isocinética dos pés de Homens Adultos Normais [dissertion]. São Paulo, Brazil: Universidade de São Paulo; 1994.

22. Winett RA, Carpinelli ED. Potential health-related benefits of resistance training. Prev Med. 2001;33(5):503-513.

23. Kraemer WJ, Adams K, Vafarelli E, et al; American College of Sports Medicine. American College of Medicine position stand. Progression models in resistance training for healthy adults. Med Sci Sports Exerc. 2002;24(2):364-380.

24. Islam MM, Nasu E, Rogers ME, Koizumi D, Rogers N1, Takeshima N. Effects of combined sensory and muscular training on balance in Japanese older adults. Prev Med. 2004;39(6):1148-1155.

25. Nagy E, Feher-Kiss A, Márnai B, Domján-Preszner A, Angyan L, Horvath G. Postural control in elderly subjects participating in balance training. Eur J Appl Physiol. 2007;100:97-104.
26. Thelen DG, Schultz AB, Alexander NB, Ashton-Miller JA. Effects of age on rapid ankle torque development. J Gerontol A Biol Sci Med Sci. 1996;51:M226-M232.

27. Hess JA, Woollacott M. Effect of high-intensity strength-training of functional measure of balance ability in balance-impaired older adults. J Manipulative Physiol Ther. 2005;28:582-590.

28. Gauchard GC, Gangloff P, Jeandel C, Perrin PP. Physical activity improves gaze and posture control in the elderly. Neurosci Res. 2003;45:409-417.

29. Kronhed ACG, Moller C, Olsson B, Moller M. The effect of short-term balance training on community dwelling older adults. J Aging Phys Act. 2001;9:19-31.

30. Bellew JW, Yates JW, Gater DR. The initial effects of low-volume strength training on balance in untrained older men and women J Strength Cond Res. 2003;17:121-128.

31. Barry BK, Carson RG. The consequences of resistance training for movement control in older adults. J Gerontol. 2004;59:730-754.
Clinical Interventions in Aging

\section{Publish your work in this journal}

Clinical Interventions in Aging is an international, peer-reviewed journal focusing on evidence-based reports on the value or lack thereof of treatments intended to prevent or delay the onset of maladaptive correlates of aging in human beings. This journal is indexed on PubMed Central, MedLine, the American Chemical Society's 'Chemical Abstracts Ser-

\section{Dovepress}

vice' (CAS), Scopus and the Elsevier Bibliographic databases. The manuscript management system is completely online and includes a very quick and fair peer-review system, which is all easy to use. Visit http://www.dovepress.com/testimonials.php to read real quotes from published authors. 